\title{
La obesidad infantil en la prensa española
}

\author{
Debra WeSTALL \\ Universidad Politécnica de Valencia \\ dwestall@upvnet.upv.es
}

Recibido: $15 / 05 / 2010$

Aceptado: 22/11/2010

\begin{abstract}
Resumen
La investigación se centra en el fenómeno de sobrepeso y obesidad infantil y su representación en 120 noticias recogidas de las ediciones digitales de tres periódicos españoles (ABC, El Mundo, El País) y publicadas entre el 1 de enero y el 30 de junio del año 2008. El análisis del contenido proporcionó datos relevantes sobre 1) la distribución de las noticias por fuente y fecha, y 2) el contenido presentado durante este tiempo. Predominaron las noticias sobre la alimentación y hábitos alimenticios ( $\mathrm{n}=22$; $18,3 \%)$ y los estudios de prevalencia ( $\mathrm{n}=18 ; 15 \%)$. Los temas comunes a los tres diarios fueron los relacionados con la salud mental de los españoles en marzo, la muerte de un niño obeso de cinco años en Murcia en abril, y las dietas “milagro” en mayo.
\end{abstract}

Palabras clave: Salud pública, sobrepeso y obesidad infantil, titulares de prensa, diarios españoles.

\section{Childhood Obesity in the Spanish Press}

\begin{abstract}
This study aims to examine how the Spanish press reports on the topic of childhood overweight and obesity in a 120-item sample complied from the online editions of three major Spanish newspapers (ABC, El Mundo, El País) between 1 January and 30 June 2008. A content analysis provided valuable data related to 1 ) the frequency and distribution of news regarding childhood overweight and obesity, and 2) the contents presented during the six-month period. The topics of foods and eating habits $(\mathrm{n}=22$; $18.3 \%)$ and the incidence of childhood overweight and obesity $(n=18 ; 15 \%)$ were prevalent throughout the study period. All three daily papers published articles related to Spaniards' mental health, the death of an obese 5-year old in Murcia and "miracle" diets in the months of March, April and May, respectively.

Key words: Public Health, Childhood Overweight and Obesity, Newspaper Headlines, Spanish Press..

\section{Referencia normalizada}

WESTALL, Debra (2011): "La obesidad infantil en la prensa española”. Estudios sobre el mensaje periodístico. Vol. 17, núm. 1, págs.: 225-239. Madrid, Servicio de Publicaciones de la Universidad Complutense.
\end{abstract}

Sumario: 1. Introducción. 2. Metodología y materiales. 3. Resultados y discusión. 3.1. La presencia de noticias SO-FAT. 3.2. El enfoque científico en las noticias SO-FAT. 3.3. El enfoque sociocultural en las noticias SO-FAT. 4. Conclusiones. 5. Referencias bibliográficas.

\section{Introducción}

Este estudio se centra en el fenómeno del sobrepeso y la obesidad infantil (SO-FAT) y su representación cuantitativa y cualitativa en las noticias publicadas en 2008 en tres diarios españoles de mayor difusión y tirada (ABC, El Mundo, El País). ${ }^{1}$ El tema resulta de enorme interés debido al creciente aumento de los índices globales de SO-

\footnotetext{
${ }^{1}$ Este trabajo se ha realizado en el marco del proyecto I+D titulado "Retórica y cultura en la información periodística sobre la salud” (HUM2007-65132FILO), cofinanciado por el Ministerio de Ciencia e Innovación (España) y los fondos FEDER.
} 
FAT, su repercusión en la salud pública y sus implicaciones económicas, sociales y culturales. Según los datos publicados por la Organización Mundial de Salud (OMS), cerca de 15 millones de niños y adolescentes en toda la Unión Europea padecen obesidad (CARROQUINO, 2007). El trabajo realizado por HAUG et al. (2009) indica que en España entre un 20-25\% de los menores son obesos, mientras que la incidencia de sobrepeso entre los niños de 2 a 9 años en España es alrededor del 31\% (BRANCA et al., 2007). Debemos reflexionar sobre las consecuencias de estas cifras, ya que estos niños suelen padecer a edades más tempranas problemas relacionados con el exceso de peso, y a lo largo de su vida adulta, tienen más probabilidad de desarrollar enfermedades crónicas, que representan una carga de mayor envergadura para el sistema sanitario (CHENG, 2007). De hecho, los científicos como ARANCETA et al. (2003), responsables de los estudios nacionales sobre la prevalencia de SOFAT, advierten de la trascendencia social de esta 'epidemia' contemporánea:

La contribución de la obesidad a la aparición de enfermedades crónicas y, por tanto, su impacto en la mortalidad prematura, en la discapacidad y en el deterioro de la calidad de vida, junto con el gasto sanitario directo e indirecto que genera y las importantes dimensiones que está adquiriendo en las sociedades desarrolladas desde la edad infantil y juvenil, han hecho que el sobrepeso y la obesidad sean un importante problema de salud pública, que en la actualidad se considera de magnitud epidémica incluso en países en transición y emergentes. (ARANCETA et al., 2003: 608)

Si bien es verdad que los más pequeños en España han ido alcanzando proporciones consideradas peligrosas por expertos en la salud pública, también es cierto que la prensa escrita se ha hecho eco de esta preocupación y, en los últimos años ha tratado numerosas cuestiones referentes a la alimentación, la salud, la enfermedad y el bienestar infantil. Hay que señalar que por parte de los expertos en seguridad alimentaria y nutrición se ha defendido la necesidad de difundir en los medios de comunicación el problema de la obesidad:

Así, uno de sus objetivos, sensibilizar a la población acerca de la trascendencia sanitaria del problema de la obesidad, está ya más próximo, pues los medios de comunicación en la sociedad moderna son un canal fundamental para la obtención de información y el cambio de comportamientos por los ciudadanos. (LOBO, 2007: 439)

Sin embargo, los medios tienden a generar y transmitir la información sobre los problemas de sobrepeso y obesidad "en forma de mensajes que muchas veces no se ajustan a la realidad científica” (REMESAR, 2006: 131). Un ejemplo claro de cómo los medios pueden incidir de pleno en el conocimiento y comportamiento del público se encuentra en la destacada cobertura del tema de la anorexia, que queda manifiesto en la reciente publicación titulada Medicina y salud en la prensa diaria - Informe Quiral 1997-2006:

En su faceta más positiva, los medios denuncian e informan sobre el problema de la anorexia, divulgan las características de esta enfermedad, contribuyen a la superación de tabúes y estigmas, fomentan el debate público sobre las causas y formas de prevención, etc. En su faceta más negativa, sin embargo, se da la situación de que un importante determinante del problema de la anorexia está relacionado precisamente con los cánones de belleza, creados social y culturalmente, pero, esto sí, reafirmados, reproducidos y distribuidos a través de los medios de comunicación. (OCC, 2008: 104) 
Es obvio que la industria alimentaria ha conseguido convertir la salud y el bienestar en un fin en sí mismos y a la vez a contribuir al ambiente obesofóbico en España (Díaz-Rojo y Morant-Marco, 2007; Morant-Marco, 2010; MorantMARCo y MARTín-LóPEZ, 2010). Así que, a pesar de su carácter supuestamente objetivo, en este clima moderno, dictado por unos cánones de belleza bastante inaccesibles para la mayoría de la población, no es de extrañar que la prensa contribuya a fomentar actitudes poco favorables a los que sufren la obesidad. BONFIGLIOLI (2007) ofrece las siguientes reflexiones sobre la influencia ejercida por los medios en el ámbito sociocultural:

[...] paradoxically, the focus on obesity may also make the issue seem irrelevant to some people, stigmatise overweight people and fuel the industries which are dependent on obesogenic lifestyles but offer only short term solutions. [...] Framing obesity as a problem of individuals neglects the physical and social environmental factors which influence our activity opportunities and food and drink choices and thus drive obesity. (BONFIGLIOLI, 2007: 2-4)

Las influencias agravantes y las actitudes estigmáticas se transmiten a través del discurso periodístico, tal y como apuntaron Evans et al. (2003). Estos autores llegaron a la conclusión de que, ante la proliferación de niños obesos y de noticias sobre la obesidad en la mayoría de países desarrollados, se ha generado un lenguaje específico que refleja cómo "the body' (our bodies) are being constructed, defined, regulated and pathologised by contemporary health discourse" (Evans et al., 2003: 215). Coinciden autores españoles como CostA-SÁNCHEz (2008) y Fúster et al. (2009) al señalar que la prensa (la gallega y la madrileña, respectivamente) tiende a 'medicalizar' y 'politizar' el discurso sobre la salud y la alimentación.

Con todo eso, carecemos a fecha de hoy de investigación centrada en el tema de SO-FAT, como la que aquí se propone. El título del estudio que nos ocupa, "algo gordo”, está relacionado, desde una perspectiva sociocultural, con el tratamiento mediático de SO-FAT en España. Para ello, hemos consultado la bibliografía científico-médica más actual sobre la obesidad infantil a nivel mundial y los estudios recientes que versan sobre cómo los medios de comunicación informan de la obesidad y otras amenazas para la salud pública. Entre los trabajos que tratan la representación mediática de la obesidad, cabe resaltar las distintas consideraciones metodológicas y los diversos resultados obtenidos por académicos multidisciplinares australianos (Bonfiglioli el al., 2007; Udell y MeHTA, 2008), británicos (Boyce, 2007; Gough, 2007) y norteamericanos (BoERO, 2007; KIM y Willis, 2007; LAWRENCE, 2004). En cuanto al entorno europeo, sobresalen dos trabajos recientes. En el primero, SANDBERG (2007) denuncia la presentación de la obesidad en la prensa escrita sueca como un problema de estética, no de salud pública, lo que se reproduce en los estereotipos de género y refuerza la estigmatización de los obesos; todo ello influye negativamente en la percepción pública de la obesidad. En el segundo trabajo, HiLberT y RIED (2009) examinan 222 artículos extraídos de distintas clases de prensa escrita alemana (nacional, local y rosa [tabloide]) y proponen que el análisis pormenorizado de cada género es necesario para determinar exactamente qué noticias son las que se presentan y revelar de qué manera se lo hacen. No es sorprendente, entonces, descubrir ciertas 
similitudes entre las conclusiones expuestas por estos investigadores y las reflexiones acerca del tratamiento contradictorio de la anorexia en España, citadas anteriormente del Informe Quiral 10 años. En palabras de Javier ARANCETA, especialista en Medicina Preventiva y Salud Pública, "tenemos más información que nunca, pero más confusión que nunca” ( «Los niños obesos podrían vivir menos que sus padres», El Mundo, 28/02/2008).

El objetivo de la investigación es, pues, seguir las pautas de trabajo establecidas en la bibliografía especializada para 1) recopilar una muestra propia de noticias relacionadas con SO-FAT, extraídas de la prensa española durante el año 2008, y 2) analizar los resultados de los primeros seis meses con el fin de a) calcular las distribuciones de frecuencia de las noticias, y b) crear una tipología de los distintos temas y contenidos tratados en los titulares además de los rasgos principales del discurso informativo evidenciado en la muestra. La elección del titular como objeto de estudio se basa en el hecho de que el titular, en la mayoría de los casos, resume la información más relevante de la noticia e indica, en gran medida, el enfoque escogido por el periodista para aproximarse al tema. En palabras de FRANCESCUTTI (2009: 2), "la confección de los titulares ocupa un lugar sobresaliente en las rutinas de redacción,” ya que éstos tienen, según SANz-ÁlaVA (2007: 62), “una función catafórica, es decir, de captar la atención del posible lector. Asimismo, son enunciados de síntesis y enunciados con fuerza retórica”.

A continuación, resumiremos el plan de trabajo para este estudio inicial, cuantificaremos la presencia de las noticias SO-FAT en la prensa española entre enero y junio de 2008, y describiremos los enfoques temáticos y contenidos representados en la muestra de 120 titulares.

\section{Metodología y materiales}

En primer lugar, se estableció, para la primera etapa de esta investigación, un período de seis meses para delimitar la recogida de noticias relativas al tratamiento mediático de SO-FAT. Se realizó una extracción de noticias a partir de una primera búsqueda (02/02/2009) con las herramientas disponibles en las ediciones digitales de los tres diarios de más tirada y circulación en España (ABC, El Mundo, El País). Para ello se combinaron las variables de fecha (periodo comprendido entre el 01/01/2008 y el 30/06/2008) y expresiones clave "obesidad infantil" y "sobrepeso infantil”. Para asegurar la captura del mayor número posible de resultados, se estimó conveniente repetir la búsqueda en otras dos ocasiones (04/04/2009 y 06/06/2009) y combinar las expresiones clave con términos: "juvenil”, "adolescentes”, "jóvenes”, "niños", “obesos”. De esta manera, se recogieron más de cien textos que representan noticias de estricta actualidad e inmediatez, artículos divulgativos y reportajes, además de entrevistas y cartas al director. La decisión de recabar todo tipo de textos es debida a que éstos ofrecen agudas observaciones sobre las nuevas tendencias socioculturales y son una fuente que aporta datos de interés para conocer mejor la realidad actual del tema tratado. Así lo hicieron Westwood y Westwood (1999: 55) en su estudio de las noticias sobre la salud a lo largo de un mes en un periódico australiano, y afirmaron que a través del análisis de los distintos géneros textuales 
se puede valorar la respuesta social a los reportajes periodísticos sobre la salud. Por último, se agruparon los resultados por fuente periodística y por fecha de publicación para registrar posibles tendencias en la distribución de las noticias SOFAT durante el periodo enero-junio 2008.

En segundo lugar, se procedió a contrastar el contenido-temático anunciado en los titulares con la información abarcada en la noticia completa con el fin de descartar cualquier resultado que no estuviera claro o directamente relacionado con SO-FAT. Es el caso de la noticia con el titular "La falta de sueño reparador aumenta el riesgo de desarrollar diabetes" (abc0201), que contiene las palabras clave de la búsqueda, pero han sido empleadas en términos bastante generales y por separado: "efectos de sobrepeso", "conexiones entre la privación de sueño crónica y las anomalías metabólicas, la obesidad y los riesgos de diabetes” y "las dos variantes de la enfermedad, la infantil y juvenil, o del tipo 1; y la del adulto, o del tipo 2". Igualmente, las palabras clave "sobrepeso" y "niños" aparecían en los comentarios de los expertos citados en la noticia titulada "La comida rápida "fuerza a comer más”, según los nutricionistas” (país3005), pero se optó por excluirla al comprobar que el periodista se limitó a enumerar las conclusiones del $50^{\circ}$ Congreso de la Sociedad Española de Endocrinología y Nutrición (SEEN). Como demuestran los ejemplos extraídos de la noticia, "sobrepeso" no se asocia específicamente con los niños, y "niños” no está relacionada al sobrepeso, sino a la ingestión de yodo:

- "Si se come de forma apresurada habitualmente, puede tener una consecuencia directa en el sobrepeso", ha explicado el especialista [el investigador Carlos Diéguez, presidente de la SEEN]. (país3005)

- Además, ha agregado [el coordinador del grupo de trabajo de la SEEN sobre déficit de yodo, Federico Soriguer], "se ha constatado que un déficit moderado de yodo en los niños [...]” (país3005)

En tercer lugar, se procuró distribuir los 120 titulares que constituyeron la muestra de acuerdo con el contenido informativo principal de la noticia, basándonos inicialmente en algunas de las categorías descritas en la bibliografía interdisciplinaria (HILBERT y RIED, 2009; OCC, 2009; WANG, 2008). Se clasificaron, pues, los reportajes y artículos según su relación temática con variables como, por ejemplo, la prevalencia (población, demografía, calidad de vida); la etiología (genética o ambiente); el tratamiento y la prevención; atención o alerta sanitaria; la dietética, la nutrición, los trastornos de comportamiento alimentario; la actividad física y el sedentarismo; los daños psicológicos (bienestar, discriminación, acoso); y las patologías que afectan a los niños y adolescentes con exceso de peso (riesgos de morbimortalidad, enfermedades cardiovasculares, síndrome metabólico, discapacidades). Adicionalmente, se añadieron categorías para las noticias sobre los sucesos de actualidad (medidas e intervenciones político-sanitarias, acciones por asociaciones profesionales y las de consumidores, etc.). Por otra parte, se establecieron dos nuevas categorías que reflejaban los contenidos representados en la muestra, especialmente los promulgados por los medios de comunicación. En una se agrupaban los distintos géneros periodísticos: crónicas, cartas al editor, entrevistas personales y opiniones de columnistas, mientras en la otra se incluían 
noticias sobre el mundo del entretenimiento de ámbito cultural y popular: descripciones de programas de televisión, críticas de obras de teatro o de actuaciones musicales.

Esta amplia aproximación metodológica, y el proceso de cotejar manualmente los 120 textos, han puesto en evidencia los distintos enfoques temáticos de cada fuente y las similitudes en cuanto al conjunto de noticias de los tres periódicos. Debemos señalar que la muestra recogida para este primer estudio está limitada en cuanto a su representatividad estadística ya que contiene únicamente lo que se habían publicado los tres diarios durante los seis meses. No obstante, se pudo comprobar que las palabras clave, sobrepeso u obesidad infanto-juvenil se mencionaban de forma explícita en 50 de los titulares (41,6\%), y en las 120 noticias de la muestra, se reflejan datos actuales y relevantes para arroyar luz sobre el contenido presentado por la prensa española. En este respecto, y a través de la metodología adoptada, se descubrió un insólito "suceso de índole sanitaria" (GonzÁlez-BoRJA, 2004) que, como veremos a continuación, sirve de punto de partida para el estudio, ya que parece ser un acontecimiento único en la historia reciente de España: la muerte en Murcia en abril de un niño obeso de cinco años. Resultó ser una de las dos muertes anunciadas por los tres diarios españoles en 2008 que se relaciona directamente con la obesidad de los menores.

\section{Resultados y conclusión}

Se presentan a continuación los resultados más relevantes del análisis de 120 titulares nacionales, publicados durante los primeros seis meses de 2008, en los que se ha cuantificado la presencia de SO-FAT en los tres diarios españoles. Se han tomado en consideración como variables del análisis las fuentes, las fechas de publicación, el contraste en el enfoque temático y los contenidos representados en los titulares y noticias. Se ha propuesto una clasificación basada en el contenido-temático que se divide en dos bloques: el del enfoque científico y el del enfoque sociocultural.

\subsection{La presencia de noticias de SO-FAT en los tres diarios españoles}

El primer objetivo del trabajo era el de analizar los titulares correspondientes a los primeros seis meses con el fin de calcular las distribuciones de frecuencia de las noticias. Se aprecia una mayor presencia de noticias SO-FAT en el diario ABC $(n=52)$, que en El Mundo ( $n=37)$ o en El País ( $n=31)$, que representan el 43,3\%, $30,8 \%$ y $25,8 \%$ de la muestra, respectivamente. Se publicaron, como medio mensual, unas ocho noticias en el diario ABC, seis en El Mundo, y cinco en El País. El número total de titulares es similar para los dos trimestres analizados: de enero a marzo ( $n=62 ; 51,6 \%$ ) y de abril a junio ( $n=58 ; 48,3 \%)$, con una cierta constancia en el número de titulares publicados por $\mathrm{ABC}$ y El País durante los dos trimestres: ABC, 25 y 27; El País, 15 y 16, respectivamente. Sin embargo, se detecta una mayor variación en el número de noticias publicadas por El Mundo en los dos trimestres (22, entre enero y marzo, y 15, entre abril y junio) además de la escasa atención a SO-FAT por parte de este diario en el mes de enero $(n=2)$ y la ausencia de este tema en junio, como comentaremos a continuación. 
De enero a mayo, los tres periódicos publicaron mensualmente entre 17 y 25 artículos (14,2-20,8\%). En los dos meses de marzo y mayo unas 50 noticias en total fueron publicadas por los tres diarios, concretamente 25 textos cada mes, lo que constituye el 41,6\% del total de la muestra. En estos dos meses también se nota el contaste en la variación numérica: en el mes de marzo, se publicaron once titulares tanto en el $A B C$ como en El Mundo, pero solamente tres en El País, mientras que en el mes de mayo, se volvieron a publicar once noticias en el $A B C$, pero solamente siete noticias en El Mundo y siete más en El País. Cabe destacar que los titulares en el mes de marzo publicados en los tres diarios alertan sobre los riesgos de padecer los españoles y en particular, los obesos, una patología psiquiátrica, y en los publicados en el mes de mayo, los peligros de las dietas 'milagro', respectivamente.

Por otra parte, resulta extraño que en el mes de junio se publicaron solamente 12 noticias SO-FAT (10\% de la muestra): nueve en $A B C$, tres en El País y ninguna en El Mundo (ni siquiera en los suplementos semanales de salud del último). El descenso en el número de titulares en este mes quizá se deba a que las noticias sobre obesidad en los tres periódicos no hicieron mención específica a SO-FAT, o bien es posible que al ser el inicio del verano, las noticias se centraran en las típicas amenazas estacionales para la salud, como por ejemplo, la exposición a luz solar o las olas de calor.

\subsection{El enfoque científico en las noticias SO-FAT}

El trabajo también tenía como objetivo el de analizar los seis meses de titulares para crear una tipología de los distintos temas tratados y descubrir los rasgos principales del contenido informativo evidenciado en la muestra. Por eso, se ha detectado dos patrones de cobertura periodística en la muestra: las noticias con enfoques científicos y las noticias con enfoques socioculturales. En cuanto al primer enfoque, encontramos 68 noticias (56,7\%) que contienen información sobre los resultados publicados en estudios científicos nacionales e internacionales en torno a la salud, la enfermedad y el bienestar infantil.

En esta categoría se encuentran, primero, 22 titulares (18,3\%) que hacen referencia a la dietética y la nutrición, especialmente a estudios sobre la importancia del desayuno, los peligros de las dietas 'milagro', como se ha comentado antes, y los hábitos alimenticios de los españoles más pequeños, que se alejan cada vez más de la llamada 'dieta mediterránea', tema de bastante peso en la muestra: "Uno de cada 5 escolares no come tomate y un tercio tampoco prueba las espinacas" (abc0901) y "Un máximo de dos hamburguesas a la semana, «a cambio de más verdura»" (abc1302).

Segundo, 18 titulares (15\%) ofrecen detalles de los informes científicos sobre la incidencia de SO-FAT dentro y fuera de España. En estos titulares se destaca el uso del lenguaje comparativo y de las estadísticas para reflejar los índices de sobrepeso y/u obesidad en las distintas regiones de la península ibérica: "Los niños españoles alcanzan la talla europea e igualan el peso de los norteamericanos" (abc1605c) y "El $20 \%$ de los niños y el 15\% de las niñas son obesos” (país1006a).

Tercero, hay 14 artículos (11,7\%) sobre las enfermedades asociadas a SO-FAT, 
por ejemplo, la diabetes, enfermedad que, como la anorexia, es de las más mediática de la última década (Revuelta y Minelli de Oliveira, 2008). En el mes de abril, seis de estos titulares anuncian, mediante la representación estadística, los resultados de la Encuesta Nacional de Salud e informan sobre el riesgo de la población de sufrir trastornos mentales. De estos seis titulares, tres hacen referencia específicamente a la población femenina ("Una de cada cuatro mujeres...”); dos, a la población general ("Uno de cada cinco españoles...") y uno destaca la incidencia en la población obesa: "Cuatro de cada diez obesos padecen una patología psiquiátrica” (abc1403a).

Cuarto, son menos numerosos ( $n=8 ; 6,7 \%)$ pero no menos impactantes los titulares que anuncian una posible reducción en la esperanza de vida de los jóvenes, y cinco de estos fueron publicados en el mes de febrero en los tres diarios. El lenguaje empleado en éstos titulares enmarca de forma determinante a las noticias. Por ejemplo, en dos titulares se atribuye este deterioro prematuro de la salud a la alimentación: "La mala alimentación podría reducir la esperanza de vida de las nuevas generaciones” (abc2702) y "Nutrición y Salud. Menos años de vida por la alimentación de hoy” (mundo2702). Son dos los titulares en los cuales se alude a los progenitores y los enlaces familiares, y recurren a las condiciones causales: "Los hijos podrían vivir menos que sus padres a causa de la obesidad” (abc2802) y “Los niños obesos podrían vivir menos que sus padres»” (mundo2802). En el último ejemplo, la personificación de la obesidad resucita el carácter maligno de la epidemia: "La obesidad amenaza la esperanza de vida de los niños” (país2802).

Finalmente, solamente seis (5\%) noticias tratan específicamente sobre los beneficios del juego y la actividad física: "Los parques infantiles ayudan a la reducción de la obesidad infantil” (mundo1502). No obstante, lo que resulta más novel es la referencia a las últimas aportaciones de la tecnología: "Un prototipo de realidad virtual combina el juego y el ejercicio físico” (abc2904) y “Jugar a la Wii como terapia” (abc1603).

\subsection{El enfoque sociocultural en las noticias SO-FAT}

Mediante el análisis de contenido realizado, identificamos 52 noticias que se refieren a SO-FAT en la sociedad (43,3\% del total enero-junio 2008) y la mayoría de éstas relatan sucesos del entorno sociocultural español (n=43; o bien el 82,7\% de esta categoría). Primero, por ser un hecho inaudito en España y un acontecimiento insólito en la sociedad española, se incluyen también en esta categoría los tres titulares que anuncian, con detalles sobre su lugar de residencia y su peso específico, la muerte repentina de un niño obeso ('mórbido', según El País) de tan solo cinco años de edad, en abril de 2008:

- Fallece en Murcia un niño de cinco años aquejado de obesidad mórbida (país0404)

- Muere en Murcia un niño de cinco años que pesaba 50 kilos (abc0504)

- Fallece un niño de cinco años a causa de su obesidad (mundo0504a)

- (subtítulo): El pequeño, que vivía en La Unión (Murcia), pesaba 50 kilos, cuando lo normal a esa edad oscila entre los 18 y los 20 kilos. (mundo0504a) 
Cabe resaltar que esto es uno de los cinco temas-noticias comunes a los tres diarios a lo largo de los seis meses. Resulta extraño que, este hecho trágico y polémico, tan cercano a los lectores de España, no volviera a ser mencionado en ninguno de los periódicos en 2008.

Segundo, se registraron 10 noticias (8,3\%) que ofrecen información sobre las distintas intervenciones públicas para prevenir la obesidad infantil. En el mes de febrero, por ejemplo, aparecen noticias sobre la publicidad de los alimentos tanto en España como en Francia:

- Según el Código Paos [Código de Autorregulación de la Publicidad de los Alimentos dirigida a Menores, Prevención de la Obesidad y Salud], DEL Ministerio De SANIDAD. Los anuncios de Burger King y McDonalds, entre los que cumplen el código de Sanidad (mundo2302)

- Contra la obesidad, nada de anuncios (abc0402)

- Combatir la obesidad - Francia suprimirá los 'spots' de ciertos alimentos en horario infantil (mundo0402)

Tercero, se identificaron nueve titulares (7,5\%) que se refieren a las peticiones y advertencias lanzadas desde los colectivos de profesionales sanitarios como éste: "Los pediatras piden más plazas en centros de salud y formación continua" (abc0803). Prevalecen, no obstante, las referencias a los cursos impartidos y las jornadas celebradas con el fin de prevenir SO-FAT, tal y como se aprecia en estos cuatro ejemplos:

- Más de 35.000 alumnos han participado ya en los cursos por una dieta sana (abc1501)

- SALUD. Los secretos de una dieta sana para los más pequeños (mundo2704)

- Las jornadas de alimentación infantil llegan a 20 colegios de la provincia (abc1605a)

- No saltarse el desayuno, una de las claves para prevenir la obesidad infantil (abc2806)

Son cuatro las noticias (3,3\%) que hacen referencia a las acciones emprendidas por las asociaciones de consumidores. Tres de éstas fueron publicadas en El Mundo y en el mes de marzo, y las tres emplean un lenguaje que resalta la lucha contra la publicidad de la comida basura, debido a su significativa relación causal con SOFAT: "Alerta por la obesidad infantil - Las asociaciones de consumidores luchan contra la publicidad de comida basura” (mundo1703).

Sin embargo, lo más interesante del análisis de los titulares referentes a la sociedad es el descubrimiento de contenido relacionado con SO-FAT en géneros como las columnas personales o los editoriales, que han recibido escasa atención en la bibliografía consultada (DíAz-RoJo, 2009; LAWRENCE, 2004). Es el caso de la subcategoría de géneros periodísticos, que incluye 14 titulares (11,7\%) y se distinguen por sus rasgos más nacionales e idiosincrásicos. Estos titulares ponen de manifiesto la preocupación por SO-FAT expresada en los comentarios de periodistas y columnistas, en las entrevistas y las cartas a los directores de los periódicos.

Respecto a los comentarios de columnistas, Gustavo Duch Guillot, en su 
columna titulada, “Homer Simpson contra Popeye” (país0103), recurre al contaste de las influencias socioculturales representadas por los dos personajes de los dibujos animados y por las comidas habitualmente asociadas a cada uno para lamentar la pérdida de la dieta tradicional española, especialmente por parte de la población infantil:

Pero el equilibrio se ha roto. La dieta mediterránea se mantiene en un reducto de restaurantes de élite, los niños desconocen el sabor de los tomates y casi nunca comen espinacas. Popeye ha sido vencido por Homer Simson [sic], devorador de perritos calientes. (país0103)

En la columna de Carmelo EnCINAs, en el mes de mayo del 2008, titulado "Dietas milagro (país2405), el periodista también denuncia las dietas que predominan el mercado alimentario española, especialmente los que se promueven la denominada "operación bikini”:

Estamos en el preludio del verano y es el momento idóneo de embaucar a quien esté o se sienta gordo. Y son muchos hombres y muchísimas mujeres. Hay cálculos que cifran en un millón el número de féminas que se han puesto a régimen esta primavera en Madrid. Un millón de señoras y señoritas, entre los 16 y los 50 años, que pretenden embutirse el mismo biquini del año anterior o, si fuera posible, uno de talla menor.

En cuanto a las entrevistas publicadas en el periodo estudiado, se inciden en el tema de la alimentación actual, tal y como se aprecia en dos titulares de $A B C$. En el primero, “«La educación nutricional es la base para saber comer»” (abc1103), se cita al médico-especialista, Ignacio Jáuregui Lobero, el “coordinador jefe del Instituto de Ciencias de la Conducta, cuya actividad principal es el tratamiento de los trastornos en la conducta alimentaria”. En el segundo, Federico Argüelles Martín, presidente de la Sociedad de Nutrición Pediátrica, afirma para el titular: “"La tendencia actual es a que los niños coman mejor que antes»” (abc1605b).

Igualmente, cabe destacar las dos entrevistas, ambas publicadas en la sección "Primer plano" de El Mundo, con especialistas españolas reconocidas en el campo de la nutrición y salud infantil. En la primera se refiere a una de las causas de la obesidad y refleja los valores sociales dominantes en España, y en la segunda, apunta a las consecuencias reinantes, es decir, las enfermedades crónicas padecidas por los niños con exceso de peso:

- Etelvina SuÁREz. 'Los adultos se empeñan en querer niños gorditos' (mundo0103)

- Bonita FALKNER. 'El niño hipertenso es resultado de la obesidad creciente' (mundo0504b)

Por otra parte, también nos parece interesantes los resultados del análisis de los titulares referentes a la sociedad que pertenecen a la subcategoría denominada 'entretenimiento popular' ( $\mathrm{n}=11 ; 9,2 \%)$. La muestra analizada contiene numerosos titulares sobre los tipos de programación televisiva que trata el tema de la obesidad, en mayor o menor grado, y que ha recibido cierta atención en la prensa. Predominan los titulares referentes a programas de televisión (reality shows o documentales, entre otros) y a las preocupaciones expresadas por los periodistas y las asociaciones de consumidores. 
Primero, se encontraron dos titulares de $A B C$, "Adelgazar en vivo y en directo" (abc2101) y "María del Kilo" (abc0704b), que aluden al debate sobre la realización de programas 'realities' para obesos. El primero refiere al relanzamiento por la cadena norteamericana NBC del programa «The Biggest Looser [sic]» (El mayor perdedor), la correspondiente especial en Nueva York, Anna Grau, explica que "[los] concursantes gordísimos se someten a duros tratamientos de choque para adelgazar ante la audiencia”. Y en el segundo, el autor Alberto GARCÍA REYES, ofrece su opinión sobre el contenido del programa “«La tarde con María», de Canal Sur, hace una sección que se llama «Operación kilo» en la que se hace mofa sin ambages, y en horario de protección infantil, de quienes sufren sobrepeso" (abc0704b). Debido a sus repercusiones negativas en la opinión pública de los obesos y en la autoestima de los mismos, no es de sorprender que estas producciones hayan sido denunciadas por las asociaciones de consumidores, tal y como se anuncia en el titular de El Mundo que ofrecemos aquí: "Emitirá el programa 'cuánto quieres perder'. Los consumidores se enfrentan a Televisa por un 'reality' de obesos” (mundo0505).

En segundo lugar, los medios emiten distintos programas dedicados a promocionar alimentos saludables y ofrecer información útil sobre cómo preparar platos más sanos. Hay que señalar las dos noticias en las que se refieren a personajes célebres quienes promocionan un alimentación ‘sana', como, por ejemplo, la estrella de cine, Gwyneth Paltrow, "Los tomates no curan el cáncer” (país0401) o la duquesa de York, Sarah Fergusson, "Una duquesa entre camioneros” (abc0703). En esta línea también se registraron dos titulares que hacen mención de SO-FAT en programas de cocina: “LA SALUD EN TU COCINA'. Recetas sanas en elmundo.es” (mundo1903) y “COMIDA SANA. El Jamie Oliver francés” (mundo1205).

El tercer tipo de programación es el reportaje, que se representa con el titulo, expresado en tono algo irónico, "El placer de comer" (mundo1403c). Como se anticipa en la breve descripción del programa, se trata de dar a conocer los hábitos alimenticios de los españoles hoy en día y poner de relieve el problema de la anorexia y la obesidad infantil en nuestro país:

En esta ocasión, 'PunTODOc' se sienta a la mesa para revelar las claves de cómo comemos en España, desmenuzar las costumbres gastronómicas de familias, jubilados, profesionales o niños, y hacer una exhaustiva radiografía de hasta qué punto nuestros hábitos alimenticios hablan de lo que somos y queremos ser. Luján Argüelles conocerá de cerca el drama que deviene de la anorexia; cuando la obsesión por la comida, mata. También será testigo de otra lucha, la de una madre y su hijo contra el sobrepeso infantil. (“El placer de comer", mundo1403c)

En cuanto a estas dos últimas subcategorías, consideramos que merecen más atención por parte de los investigadores, de acuerdo con GougH (2007: 327), quien insiste en la necesidad de sondear formal y periódicamente las tendencias en el discurso de la obesidad, subrayando, en su estudio de las representaciones periodísticas de dietas masculinas en la prensa británica, la siguiente afirmación: "Since the mass media is a powerful source of information about health matters generally, media representations bear critical examination”. 


\section{Conclusiones}

En los últimos años se ha observado el aumento considerable de la tasa de sobrepeso y obesidad en los niños y adolescentes en España. Por este motivo, los tres periódicos nacionales de más tirada han ido ampliando el espacio dedicado a noticias SO-FAT, y llegaron a lo largo del año 2008 a publicar unas 250 noticias SOFAT que incluyen distintos tipos de textos y géneros. Actualmente existe una amplia bibliografía SO-FAT, pero se hecha en falta los estudios socioculturales enfocados a la realidad española y el trato periodístico de este tema. Por eso, y con el fin de determinar el contenido de las noticias que se publica en España y comprobar los enfoques que predominan en la prensa escrita española, se recogió para este estudio una muestra de seis meses (enero-junio 2008) y de 120 titulares relacionados directamente con SO-FAT, un problema de salud pública de primer orden en España.

La prensa nacional tiende a una clara dicotomía entre factores causales de SOFAT, y los titulares en la muestra ponen en evidencia lo que WANG (2008) denomina “the 'obesogenic' environment”, concepto adoptado al español por ARNAIz (2009) como "sociedades obesogénicas", para caracterizar el entorno social actual que promueve alimentos repletos de grasas y calorías en vez de fomentar la actividad física y el deporte desde edades tempranas. Las temáticas más representadas en las noticias analizadas son las que se refieren a la investigación sobre la alimentación y la nutrición infantil $(\mathrm{n}=22)$ y a los estudios de la prevalencia de SO-FAT ( $\mathrm{n}=18)$, que representan el 18,3 y 15\% del total de la muestra, respectivamente. Tanto en el mes de marzo como en el de mayo de 2008 se publicaron un total de 25 noticias sobre SOFAT, especialmente las que trataban los riesgos relacionados con los trastornos psicológicos de los españoles y los peligros de las dietas “milagro”, respectivamente.

En cuanto al contenido sociocultural en las noticias publicadas entre enero y junio de 2008, cabe resaltar los tres titulares de abril del 2008, que anuncian, con detalles explícitos, la muerte del niño murciano de cinco años que sufría obesidad. Es la primera de dos muertes atribuidas a la obesidad infantil, y anunciadas en la prensa española en 2008, y según hemos podido averiguar, es la primera vez que ha ocurrido un acontecimiento de esta índole en España. Las referencias a la obesidad que más sorprendieron, por no haber sido nombradas en la bibliografía consultada, son las hechas por los propios medios de comunicación, en particular, los comentarios de los periodistas y de sus colaboradores, y las descripciones de programas de televisión, que manifiestan los distintos enfoques que encuadran el debate social sobre la problemática de SO-FAT en España.

Consideramos que el análisis realizado ha proporcionado una serie de datos valiosos sobre las tendencias en la distribución de noticias SO-FAT en la muestra examinada y en el tipo de contenido incluido de forma habitual en los tres periódicos nacionales, aunque el carácter exploratorio de este estudio conlleva naturalmente limitaciones en cuanto a la representatividad estadística, temporal y temáticamente. Además, la perspectiva sociocultural permitirá la realización de futuros estudios para profundizar nuestra comprensión de cómo se presenta el SO-FAT en el contexto de la salud pública y cómo la prensa nacional contribuye (o no) a fomentar el ambiente obesofóbico en España, lo cual representa, en definitiva, algo gordo. 


\section{Referencias bibliográficas}

ARANCETA, Javier; PÉREZ RODRIGO, Carmen; SERRA MAJEM, Lluís, L., RIBAS BARBA, Lourdes. et al. (2003): "Prevalencia de la obesidad en España: resultados del estudio SEEDO 2000”. Barcelona, Medicina Clínica, vol. 120, núm. 16, pp. 608-612.

ARNAIZ, Mabel Gracia (2009): "La emergencia de las sociedades obesogénicas o de la obesidad como problema social”. Revista de Nutriçao (Braziliam Journal of Nutrition) . [online]. 2009, vol. 22, núm.1, Jan./Feb., pp. 5-18.

BOERO, Natalie (2007): “All the News that's Fat to Print: The American 'Obesity Epidemic' and the Media”. Qualitative Sociology, Vol. 30, number 1, pp. 41-60.

BONFIGLIOLI, Catriona M.F. (2007): Reporting obesity: a resource for journalists. Sydney, NSW: The NSW Centre for Overweight and Obesity,

BONFIGLIOLI, Catriona M.F., SMITH, Ben .J., KING, Leslye A., CHAPMAN, Simon .F. y HOLDING, Simon .J. (2007): "Choice and Voice: obesity debates in television news". The Medical Journal of Australia (Medicine and the Media Research), Vol. 187, Issue: 8, pp. 442-445.

BOYCE, Thomas (2009): “The media and obesity”. Obesity Reviews. Vol. 8, num. 1, pp. 201-205.

BRANCA, Francesco, NIKOGOSIAN, Haik y LOBSTEIN, Tim (eds., 2007): The challenge of obesity in the WHO European Region and the strategies for response - Summary. Copenhagen, World Health Organization.

CARROQUINO, María José (2007): Prevalence of excess body weight and obesity in children and adolescents. Fact Sheet 2.3. Copenhagen, World Health Organization.

CHENG, Tsung O. (2007): "Fat kids grow up to be fat adults: A lesson to be learned from China”. International Journal of Cardiology, vol. 117, pp. 133-135.

COSTA-SÁNCHEZ, Carmen (2008): "Medicina y salud en la prensa. Las noticias de salud en los principales diarios de Galicia”. Revista Latina de Comunicación Social, 63. URL: http://www.ull.es/publicaciones/latina/_2008/03/CostaSanchez.html [consultado 10/09/2009].

DÍAZ-ROJO, José Antonio (2009): "El conceptismo como recurso retórico en columnas periodísticas personales: Análisis de un tema, la obesidad”. Estudios sobre el Mensaje Periodístico, vol. 15, pp. 207-226. Madrid, Servicio de Publicaciones de la Universidad Complutense de Madrid.

DÍAZ-ROJO, José Antonio y MORANT-MARCO, Ricardo (2007): “Persuasión lingüística, salud y belleza”. Español Actual, vol. 87, pp. 27-41.

EVANS, John; EVANS, Bethan; y RICH, Emma (2003): "The only problem is children will like their chips: education and the discursive production of illhealth”. Pedagogy, Culture \& Society, vol. 11, num 2, pp. 215-240.

FRANCESCUTTI, Luis Pablo (2009): "El tiempo de los titulares. Un análisis verbal de la titulación en la prensa española durante el periodo 1980/2005”. Estudios sobre el Mensaje Periodístico, vol. 15, pp. 243-259. Madrid, Servicio de Publicaciones de la Universidad Complutense de Madrid. 
FÚSTER LORÁN, Fernando, RIBES RIPOLL, Miguel Ángel, BARDÓN, Rocío y MARINO, Eloy (2009): “Análisis cuantitativo de las noticias de alimentación en la prensa madrileña en 2006”. Revista española de documentación científica, vol.32, núm 1, pp. 99-115.

GONZÁLEZ-BORJAS, Antonia (2004): "Salud, información periodística especializada en alza”. Ámbitos, 11-12, pp. 301-310.

GOUGH, Brendan (2007): “"Real men don’t diet»: An analysis of contemporary newspaper representations of men, food and health”. Social Science \& Medicine. Vol. 64, Issue 2, pp. 326-337.

HAUG, Ellen; RASMUSSEN, Mette; SAMDAL, Oddrun: IANNOTTI, Ron et al. (2009): "Overweight in school-aged children and its relationship with demographic and lifestyle factors: results from the WHO-collaborative Health Behaviour in School-aged Children (HBSC) Study”. International Journal of Public Health, vol. 54, pp. S167-S179.

HILBERT, Anja y RIED, Jens (2009): "Obesity in Print: An Analysis of Daily Newspapers”. Obesity Facts. Vol. 2, num. 1, pp. 46-51.

KIM, Sei-Hill y WILLIS, L.Anne (2007): "Talking about Obesity: News framing of who is responsible for causing and fixing the problem”. Journal of Health Communication. Vol. 12. Issue 4, pp. 359-376.

LAWRENCE, Regina G. (2004): "Framing Obesity: The evolution of news discourse on a public health issue". The Harvard Journal of Press/Politic.Vol. 9, num. 3, pp. 56-75.

LOBO, Félix (2007): "Políticas públicas para la promoción de la alimentación saludable y la prevención de la obesidad”. Revista Española de Salud Pública. Vol. 81, núm. 5, pp. 437-441.

MORANT-MARCO, Ricardo (2010): "Reflexiones sobre lenguaje y cosmética facial”, en Español Actual.Vol. 91, pp. 147-161.

MORANT-MARCO, Ricardo y MARTÍN-LÓPEZ, María Aránzazu (2010): "Lenguaje y hermosura: un tema lingüístico que perdura", en Revista de Filología Románica. Vol. 27, pp. 263-284.

[OCC] OBSERVATORIO DE LA COMUNICACIÓN CIENTÍFICA (UPF) y FUNDACIÓN VILA CASAS (2008): Medicina y salud en la prensa diaria: Informe Quiral 1997-2006. Barcelona, Ed. Noclay.

[OCC] OBSERVATORIO DE LA COMUNICACIÓN CIENTÍFICA (UPF) y FUNDACIÓN VILA CASAS (2009): Informe Quiral 2008. Medicina, comunicación y sociedad. Barcelona, Rubes Editorial.

REMESAR, Xavier (2006): “Cómo comunicamos? Las particulares relaciones entre científicos y medios de comunicación”. Revista Española de Obesidad. Vol. 4, núm 3, pp. 131-133.

REVUELTA, Gemma y MINELLI DE OLIVEIRA, Janaina (2008): "La salud y la biomedicina en la prensa diaria. Un análisis de diez años”. Periodística, 11, pp. 55-67.

SANDBERG, Helena (2007): "A matter of looks: the framing of obesity in four Swedish daily newspapers”. Communications, vol. 32, num. 4, pp. 447-472. 
SANZ-ÁLAVA, Inmaculada (2007): El Español Profesional y Académico. Valencia, Tirant lo Blanch.

UDELL, Tuesday y MEHTA, Kaye (2008): "When two sides go to war: Newspaper reporting of 'television food advertising restrictions' as a solution to childhood obesity”. Health, Risk \& Society. Vol. 10, Issue. 6, pp. 535-548.

WANG, Youfa (2008): “Child Obesity and Health”. HEGGENHOUGEN, K. y QUAH, S. (eds): International Encyclopedia of Public Health. Amsterdam, Elsevier, pp. 590-604.

WESTWOOD, Barbara y WESTWOOD, Geoff (1999): “Assessment of newspaper reporting of public health and the medical model: A methodological case study". Health Promotion International. Vol.14, num. 1, pp. 53-64. 